\title{
EURAMET cg-18 - state-of-the-art calibration guideline for non-automatic weighing instruments
}

\author{
Klaus Fritsch ${ }^{1}$ \\ ${ }^{1}$ Mettler-Toledo GmbH, Im Langacher 44, 8606 Greifensee, Switzerland
}

\section{ABSTRACT}

This paper presents a state-of-the-art strategy for calibrating non-automatic weighing instruments (NAWIs), based on the international calibration guideline EURAMET cg-18. While existing national and legacy guidelines have only dealt with measurement uncertainty at the time of calibration, EURAMET cg-18 also provides advice for the so-called uncertainty of a weighing result, which describes the device performance during day-to-day usage. Furthermore, as a consequence of the uncertainty of a weighing result, the concept of minimum weight is introduced. The minimum weight is the smallest amount of mass that must be weighed on the device while still adhering to a predefined relative weighing tolerance requirement. The concept of minimum weight is the most important practical and quality relevant implication of NAWI calibration, as it is the prerequisite to achieve the necessary accuracy commensurate with the quality requirements of the weighing process.

\section{Section: RESEARCH PAPER \\ Keywords: Calibration; non-automatic weighing instruments; minimum weight; quality}

Citation: Klaus Fritsch, EURAMET cg-18 - state-of-the-art calibration guideline for non-automatic weighing instruments, Acta IMEKO, vol. 8, no. 3, article 3, September 2019, identifier: IMEKO-ACTA-08 (2019)-03-03

Editor: Rugkanawan Wongpithayadisai, NIMT, Thailand

Received August 3, 2018; In final form May 8, 2019; Published September 2019

Copyright: This is an open-access article distributed under the terms of the Creative Commons Attribution 3.0 License, which permits unrestricted use, distribution, and reproduction in any medium, provided the original author and source are credited.

Corresponding author: Klaus Fritsch, e-mail: klaus.fritsch@mt.com

\section{INTRODUCTION}

The calibration of non-automatic weighing instruments (NAWIs) is one of the key activities in quality control and production that must be carried out rigorously in order to accomplish the pre-defined quality attributes of the weighing process. Concerning NAWIs, many national calibration guidelines exist, most of which are based on the concepts described in the Guide to the Expression of Uncertainty in Measurement (GUM) [1]. While these guidelines are generally quite similar, they differ in their details, which makes it difficult if not impossible to develop and use one single NAWI calibration guideline on a global level. There have been recent activities in the scientific community to address this issue by creating a harmonised approach to the calibration of NAWIs that is based on an internationally recognised calibration guideline. This process is driven by EURAMET in the form of its calibration guideline EURAMET cg-18 'Calibration of Nonautomatic Weighing Instruments', which has become the most widespread guideline in this field on a global level [2]. In most European countries, it acts as a basis for accrediting calibration laboratories according to ISO/IEC 17025 [3] and was furthermore transposed into an Interamerican Metrology System
(SIM) guideline. Thus, it is frequently applied in the Americas [4]. However, it has been - until very recently - virtually unknown in Asia. This is about to change, as the first calibration laboratories have been granted accreditation based on EURAMET cg-18 in Japan, Thailand, Malaysia, Singapore, India, and Indonesia. It is expected that the guideline will become more widely applied in Asia, thus becoming an industry standard that is used on a global level.

The value of EURAMET cg-18 is furthermore substantiated, as it not only describes how to derive uncertainty at the time of calibration, but also how to estimate the so-called uncertainty of a weighing result, characterising the performance of the instrument during day-to-day usage. This approach has significant practical importance, as it facilitates the assessment of the equipment's performance against user-specific weighing tolerance requirements. One of the most important implications of the approach is the definition of the so-called minimum weight and the safe weighing range. The concept of minimum weight was added to EURAMET cg-18 in a recent revision and describes the smallest amount of mass that needs to be weighed on the instrument to achieve a relative measurement uncertainty that is smaller than the user-specific weighing process tolerance requirement. Weighing quantities that are much larger than the 
minimum weight - in the safe range of the instrument - ensures adherence to the weighing tolerance requirement. In that regard, EURAMET cg-18 serves as a standard for achieving and maintaining weighing results with the necessary accuracy as required by the quality attributes of the weighing process. It also supports compliance with applicable normative requirements as GLP, GMP, USP, food regulations, or ISO 9001.

This paper is structured as follows: After this introduction, section 2 defines the most important terms related to calibration that are described and used. The next section details the calibration procedure of EURAMET cg-18, followed by a part that describes the estimation of the standard uncertainty of the selected calibration points, and a further part on the expansion of the standard uncertainty. Section 6 provides information on the estimation of the uncertainty of a weighing result, followed by an introduction to the concept of minimum weight and safe weighing range. Finally, in the last section the major conclusions of this article are summarised.

\section{METROLOGY TERMINOLOGY RELATED TO CALIBRATION}

\subsection{Calibration}

Calibration is one of the key activities that must be performed periodically when instruments are used for quality relevant measurements. Internationally, there are many standards that stipulate this requirement, such as ISO 9001, Good Laboratory Practice (GLP), and Good Manufacturing Practice (GMP) regulations, or standards concerned with food safety. Almost everybody working in quality control and quality assurance, either in the laboratory or in the production environment, is familiar with the applicable requirements stipulated in these documents. However, there is no common understanding concerning the definition, implementation, or specific activities that comprise calibration. Let us therefore start by defining calibration.

Calibration is a set of activities carried out on a measuring instrument to understand its behaviour. This is done by establishing a relationship between known values (measurement standards) and the associated measured values (indications). The relationship consists of a deviation and its associated uncertainty. The International Vocabulary of Metrology (VIM) [5] defines calibration as follows:

'[An] operation that, under specified conditions, in a first step, establishes a relation between the quantity values with measurement uncertainties provided by measurement standards and corresponding indications with associated measurement uncertainties and, in a second step, uses this information to establish a relation for obtaining a measurement result from an indication.'

It is evident that the relationship between the known and the measured values can only be established if the associated measurement uncertainties are derived. Unfortunately, in practice, there is a widespread misconception about calibration, as many users outside of the calibration laboratory do not consider measurement uncertainty when 'calibrating' an instrument. Measurement uncertainty is defined in the GUM [1] as 'Parameter, associated with the result of a measurement that characterizes the dispersion of the values that could reasonably be attributed to the measurand.'

In simple terms, measurement uncertainty describes how far away from the true value a measurement result might reasonably be. The measurement results and the estimated uncertainty are usually documented in calibration reports or certificates. The
ISO/IEC 17025 standard specifies the general competency requirements for laboratories to carry out tests and/or calibrations. Accreditation by an accreditation body is a formal process that certifies that a calibration laboratory is competent and fulfils the requirements stipulated by ISO/IEC 17025. It is appropriate to note, at this point, that there is some misconception in the field concerning what constitutes calibration from an accredited provider. Accreditation is awarded across a fixed scope or range of capability for each discipline of calibration. Accreditation is not a 'carte blanche' for carrying out all calibrations for an instrument user who requires the service of an accredited calibration laboratory.

In short, being accredited to ISO/IEC17025 or a similar standard means that a provider has been found to be capable of working to an accepted standard, within a stated working range, as documented in his scope of accreditation at a given level of uncertainty. Therefore, calibrations can only be considered to be 'accredited' when the calibration work falls within the stated range of a calibration organisation's accredited scope.

\subsection{Adjustment}

Before detailing how weighing instruments can be calibrated and their respective measurement uncertainty estimated, it is important to emphasise another misconception about calibration: Besides being calibrated, an instrument can also be adjusted. Adjustment is defined in the VIM as follows:

'Set of operations carried out on a measuring system so that it provides prescribed indications corresponding to given values of a quantity to be measured.'

In other words, when adjusting an instrument, its indications are modified in such a way that they correspond, as far as possible, to the quantity values of the measurement standards applied. Unfortunately, many users apply the words calibration and adjustment interchangeably, incorrectly, or even randomly. Quite often, they talk about calibrating a weighing instrument when they mean adjusting it. The VIM also emphasises this point thus:

'Adjustment of a measuring system should not be confused with calibration, which is a prerequisite for adjustment. After an adjustment of a measuring system, the measuring system must usually be recalibrated.'

This statement highlights another important aspect of calibration: Before an instrument is adjusted, it must be first calibrated in order to understand and document its behaviour. This is specifically important in order not to break the traceability chain of the preceding measurements on the instrument. Equally, after an adjustment, the instrument must usually be recalibrated. Quite often, users talk about an 'as found' calibration (a calibration before any modification [adjustment] is carried out) and about an 'as left' calibration (a calibration after any necessary adjustment and/or repair has been carried out).

\subsection{Verification}

Besides calibration, measuring instruments can also be verified. Usually, instruments need to fulfil predefined requirements, quite frequently expressed as tolerances or maximum permissible errors (mpe). The VIM defines verification as follows:

'Provision of objective evidence that a given item fulfils specified requirements.' 
While calibration only establishes the relationship between measurement standards and indications ("how well the instrument performs'), verification assesses the instrument as to whether it meets specific requirements or not ('does the instrument perform well enough?'). Usually, the outcome of verification is a 'pass' or a 'fail', while calibration itself does not provide an assessment. However, in many cases, the calibration procedure is the starting point for a subsequent assessment of the results. Therefore, it is common practice to document the assessment as an annex to the calibration certificate. Tolerances or maximum permissible errors can come from a variety of sources. With respect to weighing instruments, usually the manufacturer issues specifications for each balance or scale model. International or national testing recommendations and handbooks for weighing instruments used for applications involving commercial transactions (such as OIML R76-1 [6] or NIST Handbook 44 [7]) define mpe. Furthermore, industryspecific regulations (like USP General Chapter 41 [8]) can define tolerances. However, even more importantly, the users need to specify weighing tolerances that assure that the instrument performs well enough that they are able to fulfil their specific process requirements. In respect to the weighing application, these are the most important tolerances, as they have a direct impact on the quality of the final product.

\section{CALIBRATION PROCEDURE OF EURAMET CG-18}

Usually, a repeatability test, a test for errors of indication, and an eccentricity test are performed to assess the performance of the weighing instrument. Concerning assessment of the normal use of the instrument or evaluation of the performance under special conditions of use, EURAMET cg-18 allows for flexible execution of the tests; however, adherence to specific minimum requirements in the tests is stipulated as explained in the following paragraphs.

\subsection{Repeatability test}

Usually, a test load of about $0.5 \cdot$ Max to Max is quite common (Max refers to the maximum capacity of the instrument). However, this test load is often reduced for instruments for which the test load would amount to several thousand kilograms. For multiple range and multi-interval instruments, a load below and close to the capacity of the range/interval with the smallest scale interval $d$ may be sufficient. A special value for the test load may be agreed where this is justified in view of a specific application of the instrument. An example would be weighing standards or samples on analytical and micro-balances for which the typical quantity that is weighed is at the low end of the measurement range. Here, a small repeatability test load at the lower end of the weighing range may be agreed. The test load should, as far as possible, consist of one single body. The load must be applied at least three times when it weighs $100 \mathrm{~kg}$ or more and at least five times for smaller loads. Note that it is general practice to exceed the minimum requirement of EURAMET cg-18 for analytical and micro-balances by carrying out ten repeated weighings in order to obtain a more robust characterisation of the instrument's behaviour. This is done because for these instruments, repeatability is typically a very large contribution factor to the standard uncertainty.

Repeatability is quantified by calculating the standard deviation of the repeated measurements: $s=\sqrt{\frac{1}{n-1} \sum_{i=1}^{n}\left(I_{i}-\bar{I}\right)^{2}}$,

with $n$ being the number of repeated weighings, $I_{i}$ representing the individual indications, and $\bar{I}$ being the mean value of the indications.

\subsection{Test for errors of indication}

This test requires at least five test points, distributed fairly evenly over the weighing range of the instrument. Note that zero is considered a test point. This is because measurement uncertainty can be allocated to zero. Consequently, an error of indication test with four physical test loads, plus the zero, fulfils the minimum requirements of EURAMET cg-18.

The individual errors of indications $E_{j}$ are calculated as

$E_{j}=I_{j}-m_{r e f, j}$.

$I_{j}$ represents the individual indications of the error of indication test points. The reference value of mass $m_{r e f, j}$ of the test loads is usually approximated to its nominal value, $m_{N, j}$, or its conventional value, $m_{c, j}$. The conventional mass value of a body is defined in OIML D28 [9] as being equal to the mass of a standard that balances this body under conventionally chosen conditions, i.e. at the reference temperature of $20^{\circ} \mathrm{C}$, the reference air density of $1.2 \frac{\mathrm{kg}}{\mathrm{m}^{3}}$, and the reference density of the standard of $8,000 \frac{\mathrm{kg}}{\mathrm{m}^{3}}$.

\subsection{Eccentricity test}

The test is carried out with a test load of about $\operatorname{Max} / 3$ or higher. Depending on the shape of the load receptor, the number of the test points might vary. For rectangular and round platforms, usually four positions and the centre are taken as test points (see Figure 1).

From the indications $I_{i}$ obtained in the different positions, the differences $\Delta I_{e c c, i}$ are calculated as

$\Delta I_{e c c, i}=I_{i}-I_{1}$,

with $I_{i}$ representing the indications at the different positions of the load outside the centre and $I_{1}$ being the centre indication. For estimation of uncertainty, the largest $\Delta I_{e c c, i}$ (as an absolute value) is taken into account, which is abbreviated as $\left|\Delta I_{e c c, i}\right|_{\max }$.

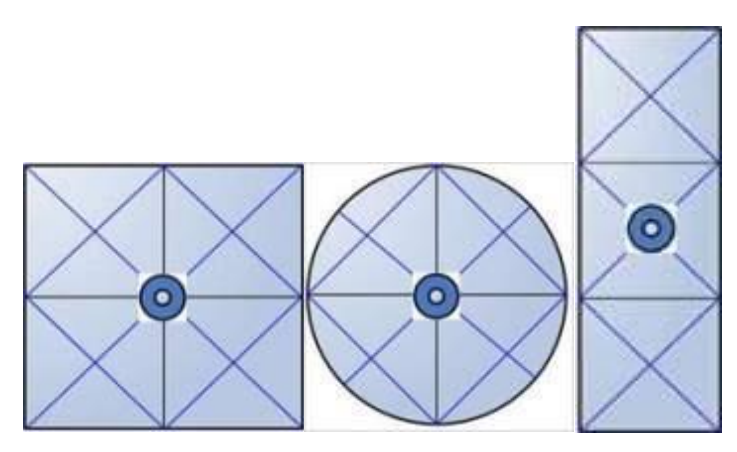

Figure 1. Graphical indication of the eccentricity test points for various platform shapes (rectangular, circular, elongated). The round circles indicate the centre, while the intersection points of the diagonals determine the offcentre positions. 


\section{STANDARD UNCERTAINTY FOR DISCRETE VALUES}

The first step in estimating the measurement uncertainty is deriving the so-called standard combined uncertainty for discrete values $u_{c}(E)$. The basic formula for that step is

$$
E=I-m_{\text {ref }} \text {, }
$$

with the related variance

$$
u_{c}^{2}(E)=u^{2}(I)+u^{2}\left(m_{r e f}\right)
$$

For better legibility, the index $j$ was omitted. Note that the above formulae apply for every error of indication test point, including zero. This means that for every error of indication test point, the associated standard uncertainty is derived individually. This is graphically indicated in Figure 2.

As can be seen from Equation (5), the standard combined uncertainty for discrete values comprises of two main sources: $u(I)$, the standard uncertainty of the indication, and $u\left(m_{\text {ref }}\right)$, the standard uncertainty of the reference mass. These two sources are also evident from the definition of calibration of the VIM, which states that the quantity values provided by the measurement standards as well as the corresponding indications are associated with measurement uncertainties.

\subsection{Standard uncertainty of the indication}

The standard uncertainty of the indication comprises four individual contributions, which account for the rounding of the no-load indication; the rounding of the indication at load; and the repeatability and the eccentricity of the weighing instrument.

\subsubsection{Rounding of the no-load indication and the indication at load}

The rounding is taken into account twice, as any indication $I$ related to a test load is the difference between the indications at load, $I_{L}$, and at no-load, $I_{0}$. The uncertainty of rounding is derived based on the assumption of a rectangular distribution of the unrounded (analogue) measurement values. In other words, it is assumed that the probability of occurrence of any unrounded measurement value (which is not indicated on the instrument) is constant within the limits of $\pm \frac{d_{0}}{2}$ or $\pm \frac{d_{L}}{2}$, respectively, where $d_{0}$ is the scale interval of the indication at zero load, and $d_{L}$ is the scale interval of the indication at load.

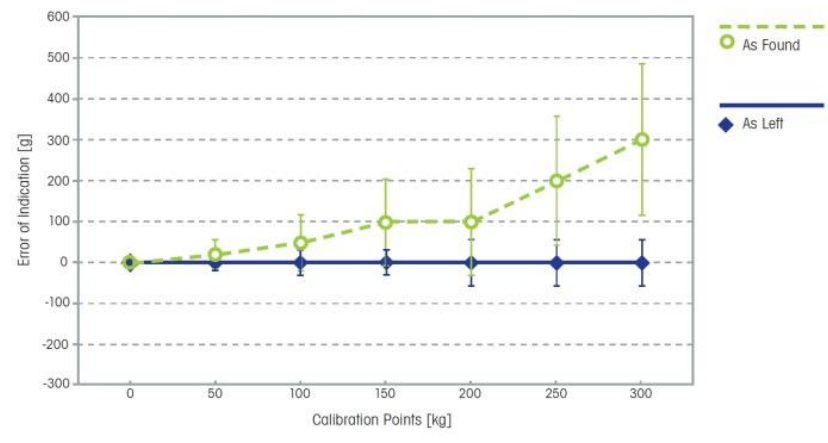

Figure 2. Graphical indication of the error of indication test points with their respective measurement uncertainty from a calibration certificate (green circles for as found [before adjustment] and blue rhombi for as left [after adjustment] of the weighing instrument). The measurement uncertainty of the as left results is typically smaller than the measurement uncertainty of the as found results as the instrument is usually adjusted in-between the two calibrations in order to optimize its performance.
The standard deviation of the rectangular distribution is $\frac{d_{0}}{2 \sqrt{3}} \approx 0.29 d_{0}$ or $\frac{d_{L}}{2 \sqrt{3}} \approx 0.29 d_{L}$, leading to the variances $\frac{d_{0}^{2}}{12}$ and $\frac{d_{L}^{2}}{12}$, respectively. In case the scale intervals at no-load and at load are the same (abbreviated as $d$ ) the total variance accounting for the rounding of the indication would thus be derived as $u_{\text {rounding }}^{2}=2 \cdot \frac{d^{2}}{12}$, leading to an uncertainty of $u_{\text {rounding }}=$ $\frac{d}{\sqrt{6}} \approx 0.41 d$. This value is also found in regulations (e.g. USP General Chapter 41) and constitutes the lowest standard uncertainty of the indication that can be realised on a weighing instrument in the case that the standard deviation of the repeatability is zero and the uncertainty related to eccentricity is neglected.

\subsubsection{Repeatability}

Normally, a single repeatability test is carried out during calibration as described in section 3.1. When estimating measurement uncertainty, the standard deviation $s$, as stated in Equation (1), is considered as being representative of all indications of the instrument and is taken as the uncertainty contribution of repeatability for all error-of-indication test points. In the case that more than one repeatability test is carried out, EURAMET cg-18 describes specific rules on how to apply the standard deviations within the weighing range(s) of the instrument. These rules are not further detailed in this article.

\subsubsection{Eccentricity}

The standard uncertainty accounting for eccentricity, $u_{\text {rel }}\left(\delta I_{e c c}\right) I$, is approximated as a linear function of the indication $I$. The relative uncertainty $u_{r e l}\left(\delta I_{e c c}\right)$ is given as

$u_{r e l}\left(\delta I_{e c c}\right)=\frac{\left|\Delta I_{e c c, i}\right|_{\max }}{2 L_{e c c} \sqrt{3}}$,

with $\left|\Delta I_{e c c, i}\right|_{\text {max }}$ being the absolute value for the largest deviation between the corner indications and the centre indication, and $L_{e c c}$ being the test load applied. Note that it is a well-established and accepted mathematical model assuming a linear relationship between the eccentric load deviation and the load applied (which is represented by the indication in the above formula). The actual behaviour of the eccentric load deviation might differ from a linear relationship based on the specific construction principle of the instrument; however, it is not common practice to assess this property at various measurement points.

\subsection{Standard uncertainty of the reference mass}

The standard uncertainty of the reference mass comprises of four individual contributions, which account for the maximum permissible error or uncertainty of the reference mass; air buoyancy; a possible drift of the reference mass since its last calibration; and convection effects due to a potential temperature difference between the reference mass and the instrument.

\subsubsection{Maximum permissible error/uncertainty of the reference mass}

Either the maximum permissible error or the uncertainty of the reference mass is taken as a contribution to the standard combined uncertainty of the weighing instrument, depending on whether the nominal or the conventional value is selected as reference mass for the calculation of the individual errors of indication. 
If the nominal value, $m_{N}$, is selected, then the maximum permissible error of the weight, mpe, is taken into account, leading to the following standard uncertainty of the reference mass

$$
u\left(\delta m_{c}\right)=\frac{m p e}{\sqrt{3}} .
$$

International recommendations and specifications such as OIML R111-1 [10] or ASTM E617 [11] define maximum permissible errors for mass standards of various accuracy classes.

If the conventional value, $m_{c}$, is selected as reference mass, then the weight uncertainty $U$ and the respective coverage factor $k$ of the weight calibration certificate are taken into account, leading to

$u\left(\delta m_{c}\right)=\frac{U}{k}$.

As both the abovementioned documents stipulate that the expanded uncertainty of the weight should not be larger than one third of the respective mpe, it is evident that the contribution of $u\left(\delta m_{c}\right)$ can be minimised if $m_{c}$ is selected instead of $m_{N}$.

In the case that a test load consists of more than one standard weight, the standard uncertainties are added arithmetically, not by sum of squares, to account for the assumed correlations of the weights.

\subsubsection{Buoyancy}

In general, air buoyancy can be corrected if the density of the reference mass, $\rho$, and the air density at the time of calibration of the weighing instrument, $\rho_{a}$, are known. Normally, however, the air density is not measured during the calibration of the weighing instrument, so an air buoyancy correction is not frequently carried out in practice. In these cases, the (unknown) buoyancy correction is taken into account as an intrinsic part of the buoyancy uncertainty.

In this scenario, two cases must be distinguished: one in which the sensitivity of the instrument is adjusted immediately before calibration and the other in which the instrument is not adjusted before calibration. If the instrument is adjusted immediately before calibration, the respective buoyancy uncertainty is smaller, as the potential change in buoyancy due to different air densities at calibration and adjustment is removed. If the instrument is adjusted independent of the calibration, worst-case assumptions should be made regarding the potential air density variation between adjustment and calibration.

If conformity of the standard weights with OIML R111-1 is established, recourse can be taken to section 10 of OIML R1111 , which stipulates that the density of the weights should be close enough to the reference density of $8,000 \frac{\mathrm{kg}}{\mathrm{m}^{3}}$, so that a deviation of $10 \%$ from the reference air density $\left(1.2 \frac{\mathrm{kg}}{\mathrm{m}^{3}}\right)$ does not produce an error attributable to buoyancy that exceeds one quarter of the weight mpe. Using this approach, the uncertainty due to buoyancy is estimated as one quarter of the uncertainty contribution of the weight itself, as expressed in Equation (7). Therefore, if the sensitivity of the instrument is adjusted immediately before calibration, the uncertainty due to air buoyancy can be derived as

$u\left(\delta m_{B}\right) \approx \frac{m p e}{4 \sqrt{3}}$, where mpe is the maximum permissible error defined by OIML R111-1 for the selected weight. If the sensitivity of the instrument is not adjusted before calibration (thereby assuming a worst-case air density variation of $10 \%$ of the reference density of air $\rho_{0}$ between adjustment and calibration), then

$u\left(\delta m_{B}\right) \approx \frac{0.1 \frac{\rho_{0}}{\rho_{c}} m_{N}+\frac{m p e}{4}}{\sqrt{3}}$,

where $\rho_{0}$ is the reference density of air, $1.2 \frac{\mathrm{kg}}{\mathrm{m}^{3}}$, and $\rho_{c}$ is the reference density of a standard weight, $8,000 \frac{\mathrm{kg}}{\mathrm{m}^{3}}$.

The uncertainty due to buoyancy is typically in the order of a few ppm of the reference mass but strongly depends on whether the instrument was adjusted before calibration or not. When the instrument was not adjusted, the uncertainty due to buoyancy could be a factor of three or more higher compared to when the instrument was adjusted before calibration.

Equation (10) constitutes a highly conservative approach to estimating uncertainty due to buoyancy, and if there is evidence that air density variations are smaller than $0.1 \rho_{0}$, this value should be substituted by a less conservative value. This is usually done by assuming realistic air pressure, temperature, and humidity variations at the installation location over the time of use of the weighing instrument, from which the respective air density variation can be calculated.

\subsubsection{Drift of the reference mass}

A possible drift of the reference mass $m_{c}$ since its last calibration can be assumed based on the difference in $m_{c}$ evident from the consecutive calibration certificates of the standard weights. The drift may be also estimated in view of the quality of the weights, as well as the frequency and care of their use, to a multiple (expressed by a factor $k_{D}$ ) of their expanded uncertainty $U\left(\delta m_{c}\right)$. It is not advisable to apply a correction to the reference mass; rather, it includes the potential drift in the uncertainty instead:

$u\left(\delta m_{D}\right)=\frac{k_{D} U\left(\delta m_{c}\right)}{\sqrt{3}}$,

$k_{D}$ is usually chosen between one and three.

For weights conforming to OIML R111-1 or ASTM E617, an upper limit $\frac{m p e}{\sqrt{3}}$ for the uncertainty contribution due to the drift of the reference mass can be applied, provided that subsequent weight calibrations confirm that the mpe of the applicable weight class is adhered to.

\subsubsection{Convection effects}

In the case that weights have been transported to the calibration site, they may not have the same temperature as the instrument and its environment. A temperature difference leads to a change in the apparent mass of the weights, $\Delta m_{\text {conv }}$, due to viscous friction at their surface induced by air flow originating from convection (Figure 3). Note that the term "apparent mass" is not a strict metrological term and not defined in OIML D28. In the context of the uncertainty of the reference mass, the apparent mass describes the fact that the gravitational force on the standard may be altered if a temperature difference between the standard and the environment in close vicinity to the instrument is present. If the calibration weight is warmer than the environment, the air that is in contact with the weight warms up and moves upward, and by means of viscous friction, its 


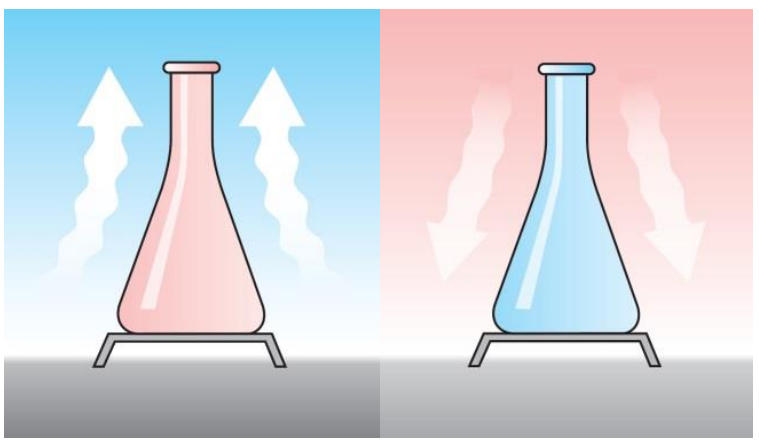

Figure 3. The effect of convection, demonstrated with an Erlenmeyer flask: In the left picture, the flask is warmer than the environment, leading to an upward convection, and in the right picture, the beaker is colder than the environment, leading to a downward convection.

apparent mass is reduced. If the weight is colder than the environment, the downward moving air increases its apparent mass.

This effect should be taken into account either by allowing the weights to acclimatise to the extent that the remaining change $\Delta m_{\text {conv }}$ is negligible in view of the uncertainty of the calibration or by considering the possible change of indication in the uncertainty budget. The effect may have to be considered for weights of a high accuracy class as OIML $\mathrm{E}_{2}$ or $\mathrm{F}_{1}$ weights. It is not advisable to apply a correction; rather, one should include the potential convection effects in the uncertainty instead:

$u\left(\delta m_{\text {conv }}\right)=\frac{\Delta m_{\text {conv }}}{\sqrt{3}}$.

Appendix F of EURAMET cg-18 provides further information and tables for deriving $\Delta m_{\text {conv }}$.

\subsection{Summary - Standard uncertainty for discrete values}

Summarising the contributions as described above, the standard combined uncertainty for discrete values, $u_{c}(E)$, can be derived as

$$
\begin{aligned}
u_{c}^{2}(E)= & \frac{d_{0}^{2}}{12}+\frac{d_{L}^{2}}{12}+s^{2}(I)+u_{r e l}^{2}\left(\delta I_{e c c}\right) I^{2} \\
& +u^{2}\left(\delta m_{c}\right)+u^{2}\left(\delta m_{B}\right)+u^{2}\left(\delta m_{D}\right) \\
& +u^{2}\left(\delta m_{c o n v}\right)
\end{aligned}
$$

Some of the factors contributing to the standard uncertainty might be relatively small, depending on the instrument and the accuracy class of the reference masses under consideration. It is within the responsibility and the competence of the calibration laboratory to neglect these contributions if appropriate.

\section{EXPANDED UNCERTAINTY AT CALIBRATION}

After having derived the standard combined uncertainty at calibration, this uncertainty must be expanded by the coverage factor, $k$, which is chosen in a way such that the expanded uncertainty of measurement has a coverage probability of $95.45 \%$ :

$$
U(E)=k u_{c}(E) .
$$

This means that the expanded uncertainty shall ensure that the true value, which is unknown, has a probability of at least $95.45 \%$ within the interval 'measured value \pm expanded measurement uncertainty'.
In the case that a normal (Gaussian) distribution can be attributed to the error of indication and the standard uncertainty is sufficiently reliable, $k=2$ applies. Normal distribution may be assumed where several uncertainty components, each derived from 'well-behaved' distributions (normal, rectangular, or similar) contribute to $u_{c}(E)$ in comparable amounts. This is normally the case when calibrating NAWIs. Sufficient reliability can be assumed, such as in the case that during a repeatability test, a load is applied no less than ten times.

In the case that a normal distribution can be attributed to the error of indication but $u_{c}(E)$ is not sufficiently reliable, then the coverage factor is determined mathematically according to the concepts described in Appendix B of EURAMET cg-18 and GUM. Such a situation usually arises when a load is applied less than ten times during the repeatability test. Due to the smaller number of the repeated weighings, the coverage factor is larger than two. The coverage factor depends on the effective degrees of freedom, $v_{\text {eff }}$, determined using the Welch-Satterthwaite formula:

$v_{e f f}=\frac{u^{4}(E)}{\sum_{i=1}^{N} \frac{u_{i}^{4}(E)}{v_{i}}}$,

where $u_{i}(E)$ refers to the contributions to the standard combined uncertainty, $u_{c}(E)$, and $v_{i}$ represents the effective degrees of freedom of the standard uncertainty contribution, $u_{i}(E)$. The coverage factor is subsequently calculated using the $t$-distribution or the student's distribution.

\section{UNCERTAINTY OF A WEIGHING RESULT}

\subsection{Interpretation of calibration data}

After calculating the expanded uncertainty, the calibration itself is formally completed. However, the data derived so far does not include the following information:

1) The behaviour of the instrument in between the selected error of indication test points;

2) The estimation of the measurement uncertainty in normal usage; and

3) The assessment of the instrument against specific requirements, such as weighing process tolerances or specifications.

The calibration data offers information only for a limited amount of error-of-indication test points. Selecting more errorof-indication test points does not resolve this problem, as this procedure still constitutes a limited picture of the real behaviour of the instrument and increases the time (and cost) of calibration. Furthermore, calibration only assesses the behaviour of the instrument at the time of calibration, but not during normal usage. Any potential influence that occurs before or after the calibration cannot be taken into account during the calibration itself. However, when assessing the instrument against specific requirements (such as weighing process tolerances or specifications), the normal usage should be taken into account as far as possible.

EURAMET cg-18 offers detailed information on how to interpret calibration data, specifically concerning how to derive the so-called standard uncertainty of a weighing result, $u_{c}(W)$. The standard uncertainty of a weighing result takes into account the normal usage of the instrument and allows for the estimation of the measurement uncertainty for any quantity of material that is placed on the weighing instrument during day-to-day use. The 
uncertainty of a weighing result is frequently used for the assessment against weighing process requirements (tolerances); for example, in respect to minimum weight. This specific assessment is detailed later in this paper.

The contributions to $u_{c}(W)$ are usually either constant over the measurement range or depend linearly on the reading of the instrument, $R$, and thus may be grouped in two terms, $\alpha_{W}^{2}$ and $\beta_{W}^{2}$

$$
u_{c}^{2}(W)=\alpha_{W}^{2}+\beta_{W}^{2} R^{2} .
$$

For multi-interval and multiple range instruments, the uncertainty of a weighing result is expressed per interval/range.

Note that the uncertainty of a weighing result is not covered by the accreditation as it is based on an interpretation of the calibration results and is not directly and exclusively derived from the measurement values of the instrument, which were taken during calibration.

\subsection{Expanded uncertainty of a weighing result} by

The expanded uncertainty of a weighing result is determined

$$
U(W)=k u_{c}(W) \text {. }
$$

For $U(W)$, the coverage factor, $k$, is equal to two in most cases, even where the standard deviation, $s$, is obtained from only a few repeated weighings and/or where $k>2$ (at calibration) is stated in the calibration certificate. This is due to the large number of terms contributing to $u_{c}(W)$.

\subsection{Errors included in uncertainty - the 'global uncertainty'}

It is common practice and could even be considered crucial to derive a 'global uncertainty', $U_{g l}(W)$, which includes the errors of indication such that no correction needs to be applied to the reading of a weighing result. In practice, applying corrections to the day-to-day work is inefficient and almost impossible. Note that the concept of 'global uncertainty' as derived in EURAMET cg-18 is not a strict metrological concept, and the name might be misleading if not referenced properly. As explained above, the 'global uncertainty' is usually derived by adding the absolute of the error of indication to the expanded uncertainty of a weighing result. This ensures that the 'global uncertainty' associated with a specific weighing process that is carried out during normal usage of the instrument establishes a sufficiently high confidence level that the mass that is weighed lies within the interval 'measured value \pm global uncertainty', without correction of the measured value.

Therefore, the weighing result in its final form can be expressed as

$W=R \pm U_{g l}(W)$

where $R$ is the reading on the device, and $U_{g l}(W)$ is the 'global uncertainty'.

A common approach to calculating the global uncertainty is to arithmetically add the expanded uncertainty of a weighing result, $U(W)$, and the absolute value of the error of indication, $E(R)$, reflecting possible correlations between these two terms. $E(R)$ is quite often approximated by a linear equation: $E(R)=$ $a_{1} R$, with $a_{1}$ being the linear regression coefficient, such that

$U_{g l}(W)=k \sqrt{\alpha_{W}^{2}+\beta_{W}^{2} R^{2}}+\left|a_{1}\right| R$
In order to facilitate the understanding and interpretation of the results, $U(W)=k \sqrt{\alpha_{W}^{2}+\beta_{W}^{2} R^{2}}$, which is a rather complicated formula, is approximated by a linear equation, such that

$$
\begin{aligned}
U_{g l}(W) & \approx U(W=0) \\
& +\frac{U(W=\operatorname{Max})-U(W=0)}{\operatorname{Max}} R+\left|a_{1}\right| R .
\end{aligned}
$$

By introducing the parameters $\alpha_{g l}$ and $\beta_{g l}$, the 'global uncertainty' can be expressed as

$U_{g l}(W)=\alpha_{g l}+\beta_{g l} \cdot R$

with the intercept $\alpha_{g l}$ (i.e. the uncertainty without load) and the slope $\beta_{g l}$ (i.e. the parameter describing the increase of the uncertainty when larger loads are applied on the instrument). The corresponding formulae for multi-range and multi-interval instruments are derived accordingly.

In the next section of this article, unless otherwise noted, uncertainty always refers to global uncertainty. The term 'global', the indexes ' $g l$ ', and the symbol ' $W$ ' are omitted to increase legibility.

\section{MINIMUM WEIGHT AND SAFE WEIGHING RANGE}

Due to its importance in practice, the concept of minimum weight was included in EURAMET cg-18 in a recent revision. 'Minimum weight' is defined in the literature as '...the smallest sample quantity required for a weighment to just achieve a specified relative accuracy of weighing' [12], [13].

It is general practice for users to define specific requirements for the performance of a weighing instrument (User Requirement Specifications). Concerning accuracy, a requirement is normally expressed as an upper threshold for the relative measurement uncertainty $U_{\text {rel }}=U / \mathrm{m}$ that is acceptable for a specific weighing application, and it is referred to as weighing process accuracy or a weighing tolerance requirement. Consequently, for a given relative tolerance requirement, TOL, only weighments with a load $m$ fulfilling the relative uncertainty inequality,

$\frac{U}{m} \leq T O L$,

comply with the respective user requirement. The limit value $m_{\min }=U / T O L$, i.e. the smallest load that fulfils the requirement, is called minimum weight. Figure 4 visualises the relative measurement uncertainty $U_{r e l}$ as a function of the applied load by using the model in Equation (21). It is evident that the smaller the load is, the larger the relative measurement uncertainty becomes, and for quantities smaller than the minimum weight, the relative measurement uncertainty exceeds the weighing tolerance requirement.

As the measurement uncertainty may be difficult to estimate due to environmental changes (vibrations, draughts, and operator influence) or specific influences of the weighing application (electrostatically charged samples, magnetic stirrers), a safety factor $S F$ that is larger than one is usually applied, such that the tolerance requirement divided by the safety factor acts as the applied user requirement:

$\frac{U}{m} \leq \frac{T O L}{S F}$. 


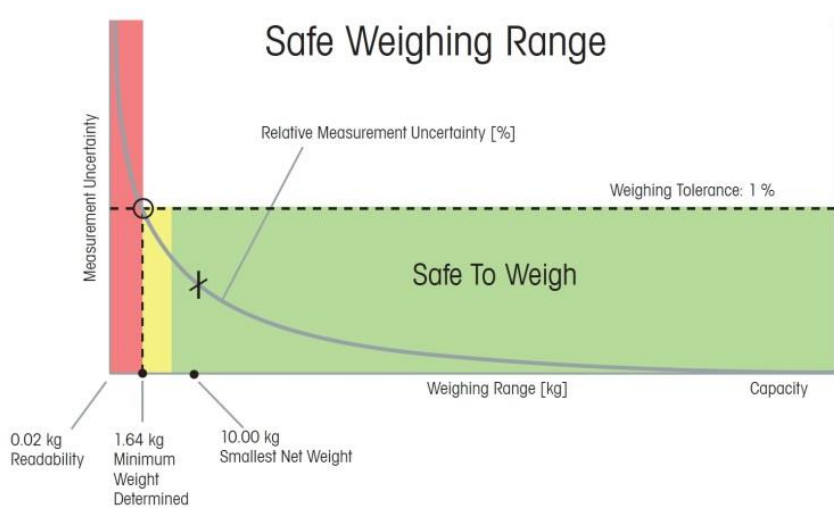

Figure 4. Safe weighing range for an industrial scale calculated from the calibration data and presented in an annex to a calibration certificate. The $x$ axis denotes the mass of the object applied on the weighing pan, while the $y$ axis denotes the associated relative measurement uncertainty in percentage. Further to the required weighing tolerance of $1 \%$, the smallest net weight of $10.00 \mathrm{~kg}$ is the mass of the smallest sample that the user intends to weigh on the balance on a day-to-day basis. In this example, the smallest net weight is in the safe weighing range, i.e. it is larger than the minimum weight including the safety factor. Thus, the calibration results have shown that the instrument fulfils the user requirement.

Consequently, using Equation (21), the minimum weight (including safety factor $m_{\text {min,SF }}$ ) can be calculated as follows:

$m_{\min , S F}=\frac{\alpha \cdot S F}{T O L-\beta \cdot S F}$.

This calculation leads to the definition of the safe weighing range: the range of the instrument, where the user fulfils the weighing tolerance requirement and takes into account the defined safety factor. The lower boundary of the safe weighing range is given by $m_{\min , S F}$. As shown in Figure 4, weighing quantities in the red region results in non-compliance with the tolerance requirement, while weighing quantities in the green region ensures the user requirement is fulfilled (safe weighing range). Weighing quantities in the yellow area fulfils the tolerance requirement; however, the safety factor is not taken into account. The smallest quantity that the user wants to weigh on the device on a day-to-day basis is called the smallest net weight. With that, we can state: When the smallest net weight equals or is larger than the minimum weight (including the safety factor [i.e. when the smallest net weight is in the safe weighing range]), then the user requirement is fulfilled.

The minimum weight and the safe weighing range refer to the net (sample) mass that is weighed on the instrument, i.e. the tare vessel mass must not be considered as fulfilling the tolerance requirement. Therefore, minimum weight is frequently called 'minimum sample weight'.

It is important to note that for weighing small loads on analytical and micro-balances, the dominant factor that contributes to the uncertainty stems from repeatability. As for these laboratory applications, the minimum weight is typically a very small sample size compared to the capacity of the balance. The absolute uncertainty at the minimum weight can be approximated by $\alpha$, and the increase of uncertainty with mass that is characterised by $\beta$ can be neglected. Furthermore, $\alpha$ mainly consists of the uncertainty contributions due to the rounding error of the indication and repeatability, with repeatability being the dominant factor for such types of instruments (the uncertainty due to eccentricity errors is usually very small compared to the uncertainty from rounding and repeatability). Consequently, the minimum weight for applications when small loads are weighed on analytical and micro-balances (for a safety factor of 1 ) can be approximated as

$m_{\min }=\frac{\alpha}{T O L-\beta} \approx \frac{\alpha}{T O L} \approx \frac{k \cdot s}{T O L}$.

An example of this simplified approach to minimum weight is presented in USP General Chapter 41. In this regulation, the repeatability requirement is defined as:

'Repeatability is satisfactory if two times the standard deviation of the weighed value, divided by the desired smallest net weight, does not exceed $0.10 \%$.'

In other words, repeatability is considered the only significant contribution to the standard uncertainty. This approach is valid for the affected weighing applications, as typically analytical and micro-balances are used to weigh small quantities of samples or standards. In both USP General Chapters 41 and 1251 [14], the concept of minimum weight as described above is included. Taking the repeatability criterion of USP General Chapter 41, the minimum weight is derived as

$m_{\min }=\frac{k \cdot s}{T O L}=\frac{2 \cdot s}{0.10 \%}=2000 \cdot s$.

\section{CONCLUSIONS}

The calibration of measuring instruments is among the most important activities within any quality management system. Unfortunately, industry practices with respect to weighing instruments do not always appropriately reflect state-of-the-art concepts. The most evident shortcoming was the lack of a scientifically correct estimation of the measurement uncertainty, which is needed to assess whether the instrument under consideration fulfils the predefined process tolerances. The EURAMET cg-18 calibration guideline is the most widespread reference document that details the methodology of deriving the measurement uncertainty of non-automatic weighing instruments. It not only includes information concerning uncertainty at calibration, but it also includes information on the uncertainty of a weighing result that describes the performance of the instrument during day-to-day work. The guideline frequently serves as a basis for assessing an instrument against predefined tolerances. A critical consequence of calibration is the concept of the minimum weight. By determining the minimum weight, a user can assure compliance with their weighing requirements by weighing a sufficiently higher quantity of material than the minimum weight, expressed quantitatively by the safety factor. The minimum weight under consideration of the safety factor defines the lower boundary of the safe weighing range and weighing quantities of material within the safe weighing range ensures compliance with the required weighing process tolerance. In simple words, calibration and the subsequent interpretation of its data establishes the minimum weight and the safe weighing range, and it ensures that the user meets the applicable quality requirements.

\section{REFERENCES}

[1] JCGM, 'Evaluation of measurement data - Guide to the expression of uncertainty in measurement', JCGM 100, 2008.

[2] EURAMET, 'Guidelines on the calibration of non-automatic weighing instruments', EURAMET cg-18, 4th ed., 2015. 
[3] ISO/IEC, 'General requirements for the competence of testing and calibration laboratories', ISO/IEC 17025, 2017.

[4] SIM, 'Guidelines on the calibration of non-automatic weighing instruments', 2008.

[5] JCGM, 'International vocabulary of metrology - basic and general concepts and associated terms', JCGM 200, 3 $3^{\text {rd }}$ ed., 2012.

[6] OIML, Non-automatic weighing instruments, Part 1: Metrological and technical requirements - Tests', OIML R76-1, 2006.

[7] NIST, 'Specifications, tolerances, and other technical requirements for weighing and measuring devices', NIST Handbook 44, 2018.

[8] USP, 'Balances', USP General Chapter 41, US Pharmacopeia USP42 - NF37, 2019.

[9] OIML, 'Conventional value of the result of weighing in air', OIML D28, 2004.
[10] OIML, 'Weights of classes $\mathrm{E}_{1}, \mathrm{E}_{2}, \mathrm{~F}_{1}, \mathrm{~F}_{2}, \mathrm{M}_{1}, \mathrm{M}_{1-2}, \mathrm{M}_{2}, \mathrm{M}_{2-3}$ and $\mathrm{M}_{3}$, Part 1: Metrological and technical requirements', OIML R111$1,2004$.

[11] ASTM, 'Standard specification for laboratory weights and precision mass standards', ASTM E617, 2018.

[12] R. Nater, A. Reichmuth, R. Schwartz, M. Borys, P. Zervos, Dictionary of Weighing Terms - A Guide to the Terminology of Weighing, Springer Berlin Heidelberg, 2009, ISBN 978-3-64202013-1.

[13] K. Fritsch, 'GWP® - The science-based global standard for efficient lifecycle management of weighing instruments', NCSLI, Measure J. Meas. Sci. 8, 3 (2013) pp. 60-69.

[14] USP, 'Weighing on an analytical balance', USP General Chapter 1251, US Pharmacopeia USP42 - NF37, 2019. 